\title{
The retinoid anticancer signal: mechanisms of target gene regulation
}

\author{
T Liu', A Bohlken', S Kuljaca', M Lee', T Nguyen', S Smith', B Cheung', MD Norris', M Haber', AJ Holloway², \\ DDL Bowtell ${ }^{2}$ and GM Marshall*,1,3
}

'Children's Cancer Institute Australia for Medical Research, Randwick NSW 203I, Australia; ²Peter MacCallum Cancer Centre, East Melbourne VIC 8006, Australia; ${ }^{3}$ Centre for Children's Cancer and Blood Disorders, Sydney Children's Hospital, High Street, Randwick NSW 203I, Australia

Retinoids induce growth arrest, differentiation, and cell death in many cancer cell types. One factor determining the sensitivity or resistance to the retinoid anticancer signal is the transcriptional response of retinoid-regulated target genes in cancer cells. We used cDNA microarray to identify 3 I retinoid-regulated target genes shared by two retinoid-sensitive neuroblastoma cell lines, and then sought to determine the relevance of the target gene responses to the retinoid anticancer signal. The pattern of retinoid responsiveness for six of 13 target genes (RAR $\beta 2$, CYP26AI, CRBPI, RGSI6, DUSP6, EGRI) correlated with phenotypic retinoid sensitivity, across a panel of retinoid-sensitive or -resistant lung and breast cancer cell lines. Retinoid treatment of MYCN transgenic mice bearing neuroblastoma altered the expression of five of nine target genes examined (RAR $\beta 2$, CYP26AI, CRBPI, DUSP6, PLAT) in neuroblastoma tumour tissue in vivo. In retinoid-sensitive neuroblastoma, lung and breast cancer cell lines, direct inhibition of retinoid-induced RAR $\beta 2$ expression blocked induction of only one of eight retinoid target genes (CYP26AI). DNA demethylation, histone acetylation, and exogenous overexpression of RAR $\beta 2$ partially restored retinoid-responsive CYP26AI expression in RA-resistant MDA-MB-23 I breast, but not SK-MES-I lung, cancer cells. Combined, rather than individual, inhibition of DUSP6 and RGS 6 was required to block retinoid-induced growth inhibition in neuroblastoma cells, through phosphorylation of extracellularsignal-regulated kinase. In conclusion, sensitivity to the retinoid anticancer signal is determined in part by the transcriptional response of key retinoid-regulated target genes, such as RAR $\beta 2$, DUSP6, and RGSI 6.

British Journal of Cancer (2005) 93, 310-318. doi: I0.1038/sj.bjc.6602700 www.bjcancer.com

Published online 12 July 2005

(C) 2005 Cancer Research UK

Keywords: retinoid; retinoid signalling; gene expression profiling; neuroblastoma

Retinoids, including retinoic acid (RA), regulate the expression of genes involved in cell proliferation, differentiation, and apoptosis, and are essential for normal embryonic development and health in the adult (Evans and Kaye, 1999). The multiple phenotypic effects of retinoids are mediated, in part, by a classical pathway involving two classes of nuclear receptors: retinoic acid receptors (RARs) and retinoid X receptors (Chambon, 1996). The transcriptional activation, which follows liganded RAR binding to a RA responsive element (RARE), triggers retinoid target gene expression or suppression, and subsequent specific biological effects. In addition to the classical nuclear RA signalling mechanism, covalent binding of RA or RAR to other cellular macromolecules may exert other retinoid effects (Altucci and Gronemeyer, 2001; Balmer and Blomhoff, 2002).

Disruption of normal retinoid signalling has been causally linked to the genesis of several human and experimental cancers (reviewed in (Sun and Lotan, 2002)). Defined mechanisms of

*Correspondence: Dr GM Marshall, Centre for Children's Cancer and Blood Disorders, Sydney Children's Hospital, High Street, Randwick NSW 203I, Australia; E-mail: g.marshall@unsw.edu.au Revised 23 May 2005; accepted 13 June 2005; published online 12 July 2005 retinoid resistance in cancer cells have included increased retinoid catabolism, reduced expression of nuclear retinoid receptors, and repressed transcriptional response of RA target genes (Freemantle et al, 2003).

Following retinoid treatment in vitro, many cell types upregulate the expression of target genes coding for proteins involved in retinoid binding and metabolism, such as the nuclear $\operatorname{RAR} \beta 2$ and retinoic acid hydroxylase (CYP26A1) (Sonneveld et al, 1998; Cheung et al, 2003). The RA-responsive transcription of RAR $\beta 2$ is frequently lost in breast, lung, prostate, cervical, and oral carcinoma (Sun and Lotan, 2002; Freemantle et al, 2003). We have previously shown that derepression, or exogenous overexpression, of RAR $\beta 2$ can restore retinoid responsiveness of some cells (Cheung et al, 2003). We, and others, have also provided evidence that unliganded $\operatorname{RAR} \beta 2$ may have an additional role as a tumour suppressor gene (Houle et al, 1993; Cheung et al, 1998). However, target genes of liganded or unliganded RAR $\beta 2$ have not yet been defined. CYP26A1, on the other hand, leads to RA catabolism.

Here, we have defined a group of 31 RA-regulated genes in RA-responsive neuroblastoma cells in vitro. A subset of these target genes was also regulated in vivo, and correlated with phenotypic retinoid sensitivity in lung and breast cancer cells 
in vitro. RA-induced expression of liganded $\mathrm{RAR} \beta 2$ directly regulated CYP26A1 in RA-sensitive and -resistant neuroblastoma, lung and breast cancer cells. Promoter methylation and histone deacetylation, in part, explained the lack of retinoid responsiveness of target genes in some RA-resistant cells. Synchronous induction by RA of two target genes, known to be mitogenactivated protein kinase (MAPK) extracellular-signal-regulated kinase (ERK) signaling pathway inhibitors, was required to mediate retinoid effects on cell proliferation, through reduction of ERK phosphorylation.

\section{MATERIALS AND METHODS}

\section{Cell culture}

Human neuroblastoma BE(2)-C and SH-SY5Y cell lines were generously supplied by Dr J Biedler (Memorial Sloan-Kettering Cancer Center, NY, USA). Human lung Calu- 6 and SK-MES-1, and mammary T47D and MDA-MB-231 epithelial cancer cells were obtained from the American Type Culture Collection (Manassas, VA, USA). All cells were cultured in Dulbecco's modified Eagle's medium supplemented with L-glutamine and $10 \%$ fetal calf serum. (aRA) all-trans RA, 13-cis-RA, trichostatin A (TSA, an inhibitor of histone deacetylase) (Sigma, St Louis, MO, USA) were solubilised in ethanol. The DNA demethylation agent aza-CdR (5-aza-2deoxycytidine) (Sigma) was dissolved in water.

\section{cDNA microarray}

After treatment with control or $10 \mu \mathrm{M}$ aRA for $1,24 \mathrm{~h}, 3$ or 7 days, $\mathrm{BE}(2)-\mathrm{C}$ and SH-SY5Y cells were lysed, and RNA extracted with the standard guanidinium/phenol/chloroform method. Direct labelling cDNA microarray experiments were carried out as described previously (Boussioutas et al, 2003). Results from three independent microarray hybridisations, with probes synthesised using RNA from three independent cell treatment experiments, were analysed. An arbitrary postnormalisation cutoff of two-fold up- or downregulation was used to define significant differential gene expression.
Semiquantitative competitive reverse transcriptionpolymerase chain reaction ( $R T-P C R$ )

Confirmation of microarray data in the two neuroblastoma cell lines, in mammary and lung cancer cell lines, and in neuroblastoma tissues was carried out with competitive RT - PCR with RNAs from three independent cell culture experiments and from 12 mice. All results of RT-PCR for each gene were analysed from at least three PCR results. The competitive PCR techniques have been previously described (Norris et al, 1996; Cheung et al, 2003), which involved determining a ratio between the level of expression of a target gene and that of the house-keeping gene $\beta 2$-microglobulin $(\beta 2 \mathrm{M})$ in total RNA samples. Fold induction of a target gene by RA was calculated by ascribing the ratio as 1.0 for control-treated samples. Specific primers are listed in Table 1.

\section{Small interfering RNA (siRNA) designing}

Small interfering RNA for RAR $\beta 2$ was purchased from Dharmacon (Dharmacon Research, Lafayette, CO, USA) (Catalogue number: M003438-00-05). The sequences of the siRNA were patented and cannot be published according to Dharmacon. Small interfering RNA target sequences of 21 nucleotides for DUSP6 and RGS16 were identified using the principles described by Elbashir et al (2001). Three suitable targets were found for DUSP6: AAGAACTGTGGTGTCTTGGTA, AAGCTCAATCTGTCGATGAAC, and AAGTGCGGAATTGGTTAATAC; and three targets for RGS16: AAGATCCGATCAGCTACCAAG, AAACTTCTCAGAAGATGTGCT, and AACAAGGCAGAAAAGGATCCT. Double-stranded siRNA oligos were in vitro transcribed with Ambion Silencer siRNA Construction Kit (Ambion, Austin, TX, USA) according to the manufacturers' instructions. Scrambled siRNAs with the same GCAT content as target siRNAs, but different sequences, were also in vitro transcribed, and it was confirmed that all siRNAs did not resemble any other mRNA $(<15 / 21)$.

\section{Transient transfection}

Plasmid $c D N A$ RAR $\beta 2$ and control plasmids were kindly provided by Professor P Chambon (INSERM, Strasbourg, France).

Table I Primers for semiquantitative competitive RT-PCR for human $(\mathrm{Hs})$ cell lines and mouse $(\mathrm{Mm})$ tissues

\begin{tabular}{|c|c|c|}
\hline Gene & Forward primer & Reverse primer \\
\hline$\beta 2 M(H s)$ & ACCCCCACTGAAAAAGATGA & ATCTTCAAACCTCCATGATG \\
\hline $\mathrm{RAR} \beta 2(\mathrm{Hs})$ & СTACACTGCGAGTCCGTCTT & CAGAGCTGGTGCTCTGTGTT \\
\hline CYP26AI (Hs) & CAGCCACATCTCTGATCACT & AAGTTGTTCCAAAATTTCCA \\
\hline $\mathrm{CRBPI}(\mathrm{Hs})$ & AGGCATAGATGACCGCAAGT & TCATCTCTAGGTGCAGCTCA \\
\hline CRABPI (Hs) & GATCCACTGCACCCAAACTC & AAGCCAGCTGCCTTCATTCC \\
\hline RGSI6 (Hs) & GTGGGGCAGTAAACACAGCA & GAACTCCAGGTTCTCCTCAC \\
\hline DUSP6 (Hs) & GTTITTCCCTGAGGCCATTT & TAGGCATCGTTCATCGACAG \\
\hline $\mathrm{TIAI}(\mathrm{Hs})$ & AAGGATTTGGAGTAGATCAA & AGTCCCGGCTCACTGTGTTT \\
\hline RET (Hs) & GGAAAAGTGGTCAAGGCAAC & ATGTGGGTGGTTGACCTGCT \\
\hline FLNB $(\mathrm{Hs})$ & AGAGCATCACCCGCACCAGT & GCACAATСTCTGCСTCAGTC \\
\hline EGRI (Hs) & CAGCAGCAGCAGCACCTTCA & CGATGTGTITGGCTGGGGTA \\
\hline PLAT $(\mathrm{Hs})$ & AGGGCTGGAGAGAAAACCTC & CGAAACGAAGACTGCTCCAC \\
\hline SMAD3 $(\mathrm{Hs})$ & GTGACCACCAGATGAACCAC & GTAGTAGGAGATGGAGCACC \\
\hline DLKI $(\mathrm{Hs})$ & GTCCCCTITGTGACCAGTGC & GAGGAGCAGGCCCGAACATC \\
\hline$\beta 2 M(\mathrm{Mm})$ & TGGTGCTTGTCTCACTGACC & CGGGTGGAACTGTGTTACG \\
\hline $\operatorname{RAR} \beta 2(\mathrm{Mm})$ & ACAAGTCATCGGGCTACCAC & CAGTACTGGCATCGGTTCCT \\
\hline CYP26AI (Mm) & ACCCACATGTCСТCCAGAAA & AGGATTCAATCGCAGGGTCT \\
\hline CRBPI $(\mathrm{Mm})$ & GGACTTCAACGGGTACTGGA & AGTTGGCGATTITGCGTAAG \\
\hline DUSP6 (Mm) & AGTTITCCCTGAGGCCATT & CATCGTTCATGGACAGGTTG \\
\hline PLAT (Mm) & ACTCAGTGCCTGTCCGAAGT & GCACTGGCAGACAAAGTCAG \\
\hline RGSI 6 (Mm) & GCTCCGATACTGGGGGTATT & CGTCTITAGGAAGGCATGGA \\
\hline FLNB $(M m)$ & CCCAAACTCAACCCAAAGAA & ССTTCTGGGTCCTCAACAAA \\
\hline EGRI (Mm) & GAGCGAACAACCCTATGAGC & GGGATAACTCGTCTCCACCA \\
\hline $\operatorname{RET}(\mathrm{Mm})$ & ACAAGAGGCCAGTGTITGCT & GTGAGTCCGAAGGTGTGGAT \\
\hline
\end{tabular}


Transient transfection was performed using Superfect transfection reagent (Qiagen, Clifton Hill, Victoria, Australia) in MDA-MB-231 and SK-MES-1 cells, or Lipofectamine 2000 reagent (Invitrogen, Carlsbad, CA, USA) in SH-SY5Y cells. After $8 \mathrm{~h}$, cells were treated with $10 \mu \mathrm{M}$ aRA or control. Transfection efficiency and its effect on potential target gene expression were assessed by RT-PCR with RNA extracted $24 \mathrm{~h}$ or 3 days after aRA treatment.

Small interfering RNA SH-SY5Y, Calu-6, and T47D cells were transfected with $100 \mathrm{~nm}$ siRNA using Lipofectamine 2000 reagent according to Invitrogen. After $8 \mathrm{~h}$, cells were treated with $10 \mu \mathrm{M}$ aRA or control. RNA and/or protein were extracted $48 \mathrm{~h}$ later. Transfection efficiency and its effect on potential target gene expression was assessed by RT-PCR and/or immunoblot.

\section{Immunoblot analysis}

Cells were lysed, and protein extracted and analysed by sodium dodecyl sulphate - polyacrylamide gel electrophoresis. For DUSP6 and RGS16 studies, membranes were probed with either rabbit anti-RGS16 antibody (diluted $1: 500$ ) or goat anti-DUSP6 antibody $(1: 500)$ (Santa Cruz Biotechnology, CA, USA) followed by horseradish peroxidase (HRP)-conjugated anti-rabbit $(1: 2000)$ or anti-goat antiserum $(1: 2000)$ (Pierce, Rockford, IL, USA), respectively. The membranes were then incubated with fluorescence-conjugated ECL Plus (Amersham) and scanned with a Typhoon Scanner (Amersham). Membranes were lastly reprobed with anti- $\beta$-actin antibody (Pierce) as a loading control. Comparative protein expression was semiquantified by analysing target protein bands $v s \beta$-actin with ImageQuant software (Amersham).

For the MAPK ERK phosphorylation study, membranes were probed with mouse antiphosphorylated ERK1/2 antiserum $(1: 2000)$ (Upstate Biotech, MA, USA), followed by incubation with HRP-conjugated anti-mouse antiserum $(1: 2000)$ (Pierce). Equal loading of protein was confirmed by probing the membranes for total ERK protein with rabbit anti-ERK1/2 (nonphosphorylated) antiserum $(1: 2000)$ (Upstate Biotech), followed by incubation with HRP-conjugated anti-rabbit antiserum $(1: 2000)$ (Pierce).

\section{Cell proliferation assay}

The cell proliferation assay was carried out with the In Situ Cell Proliferation Kit, FLUOS (Roche Applied Science, Switzland), according to the manufacturer's instructions. SH-SY5Y cells transfected with scrambled siRNA, DUSP6 siRNA, RGS16 siRNA, a combination of DUSP6 and RGS16 siRNAs, or a combination of scrambled siRNAs, were treated with $10 \mu \mathrm{m}$ aRA for $64 \mathrm{~h}$, followed by incubation with $10 \mu \mathrm{M}$ 5-bromo-2'-deoxyuridine (BrdU) for $45 \mathrm{~min}$. After fixation in $70 \%$ ethanol overnight and denaturation with hydrochloric acid, cells were incubated with an anti-BrdUFLUOS antibody and analysed on a flow cytometer (FACScan, Becton Dickinson, NJ, USA). Cells incubated with solvent instead of BrdU, but incubated with anti-BrdU-FLUOS were used as control for autofluorescence and background staining. Low and high fluorescence regions were defined for quantitation: low fluorescent regions comprised $>95 \%$ of control cells (BrdUnegative or nonproliferating cells), whereas high fluorescent regions contained proliferating BrdU-positive cells. The percentage of BrdU-negative and -positive cells over total cell population was compared among target gene siRNA-, and scrambled siRNAtransfected cells treated with $10 \mu \mathrm{M}$ aRA.

\section{Animal model studies}

The generation of the MYCN transgenic mice has been described previously (Weiss et al, 1997). All homozygote MYCN transgenic mice developed neuroblastoma at the age of 6-7 weeks (Weiss et al, 1997). Once a tumour was palpable in the abdomen, homozygote MYCN transgenic mice were randomised and treated with either solvent control $(n=6)$ or 13-cis-RA at the dosages of $0.72-1.43 \mathrm{mmol}_{2} 4 \mathrm{~h}^{-1}(n=6)$ via an Alzet micro-osmotic pump (ALZET, Cupertino, CA, USA). The pump was surgically implanted subcutaneously at the back, which caused minimal discomfort to the mice (Burkhart et al, 2003). The drugs were delivered at a constant rate of $0.5 \mu \mathrm{Lh}^{-1}$ (Burkhart et al, 2003). Mice were monitored once every day from the commencement of treatment and killed 5 days after treatment. Tumour tissues were removed and RNA was extracted. Competitive RT-PCR was carried out to examine whether RA target genes identified in vitro were modulated by RA in vivo as well. All animal experimental procedures were approved by the University of New South Wales Animal Care and Ethics Committee, and were consistent with United Kingdom Coordinating Committee on Cancer Research guidelines for the welfare of animals in experimental neoplasia. Compared with untreated animals and animals treated with control, 13-cis-RA treatment did not induce any significant side effects.

\section{Statistical analysis}

All data for statistical analysis were calculated as mean \pm standard error. Differences were analysed for significance using ANOVA among groups. A probability value of 0.05 or less was considered significant.

\section{RESULTS}

\section{Microarray data and validation of a subset of differentially expressed RA target genes}

To identify RA-regulated target genes in neuroblastoma cells, we performed triplicate microarray experiments comparing gene expression in $\mathrm{BE}(2)-\mathrm{C}$ and $\mathrm{SH}-\mathrm{SY} 5 \mathrm{Y}$ neuroblastoma cell lines treated continuously with $10 \mu \mathrm{m}$ aRA for $1,24 \mathrm{~h}, 3$ or 7 days. SH-SY5Y cell line is nonamplified, and the $\mathrm{BE}(2)-\mathrm{C}$ cell line is amplified, for the oncogene $M Y C N$, an important determinant of the RA response in vitro and patient prognosis in vivo (Bordow et al, 1998; Nguyen et al, 2003).

Microarray slides with 4500 cDNA clones, representing predominantly genes with known functions, were hybridised with cDNA from aRA- or control-treated cells. In total, 31 genes were up- or downregulated by $\geqslant 2$-fold by aRA in both cell lines, at one or more time points in triplicate $(P<0.05)$ (listed in Table 2 according to the time course of changes in gene modification). This list of RA-regulated targets included genes coding for proteins known to be involved in: (i) retinoid binding and metabolism $(\mathrm{RAR} \beta 2$, cellular retinoid-binding protein 1 (CRBP1), and CYP26A1); (ii) the MAPK signalling pathway (regulator of G-protein signalling 16 (RGS16) and dual specificity phosphatase 6 (DUSP6)); (iii) cell structure and differentiation (filamin B (FLNB), $\mathrm{c}-R E T$ proto-oncogene (RET), $\alpha$ polypeptide $\mathrm{Cu}^{2+}$ transporting ATPase (ATP7A), and $\delta$-like 1 homolog (Drosophila) (DLK1)); and (iv) angiogenesis or cancer invasion (early growth response protein 1 (EGR1) and tissue plasminogen activator (PLAT)). Among the 31 genes, a classical RARE could be found in the promoter region of $\operatorname{RAR} \beta 2, \mathrm{CYP} 26 \mathrm{~A} 1, \mathrm{CRBP} 1$ and PLAT (Chambon, 1996; Sun and Lotan, 2002). While RGS16 expression was upregulated at $1 \mathrm{~h}$ after aRA (Table 2, Figure 1A), all other RA target genes showed a more gradual change in expression at 1,3 , and 7 days after commencement of aRA. Only four of 31 target genes demonstrated downregulated expression patterns after aRA treatment. In addition to those genes listed in Table 2, a further 21 genes exhibited $\geqslant 2$-fold change in expression in only one of the two neuroblastoma cell lines (data not shown). To validate the microarray data, semiquantitative, competitive RT-PCR was 
Table 2 Comparison of target gene fold induction by aRA between microarray data (outside parenthesis) and competitive RT-PCR results (inside parenthesis) in neuroblastoma cells treated with $10 \mu \mathrm{M}$ aRA

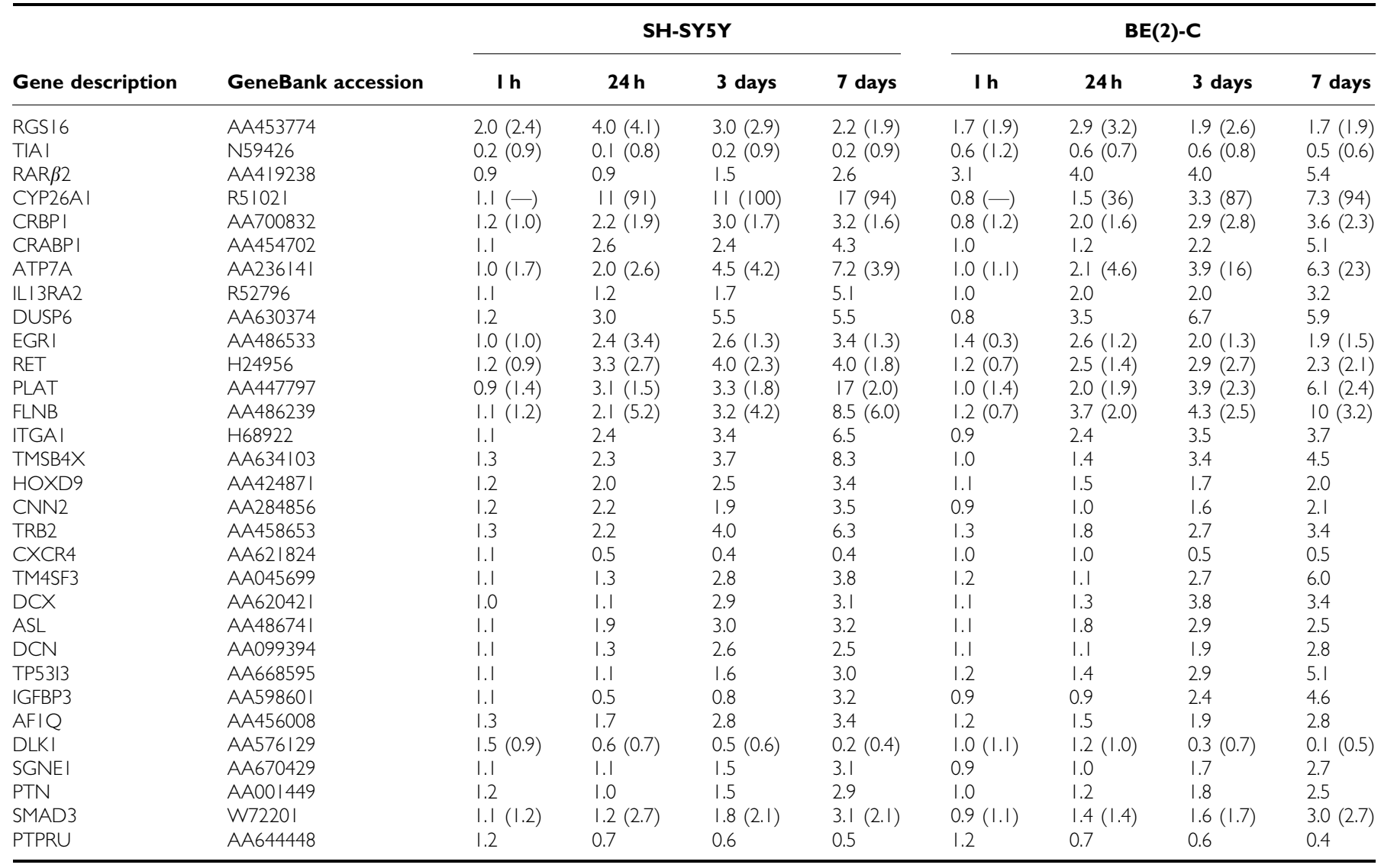

$(-)$ represents undetectable by PCR.

employed to assess expression of 11 selected genes shared by the two cell lines (Table 2). The results of RT-PCR were consistent with the microarray data in 10 out of 11 genes, with TIA1 as the only exception. Examples of the RT-PCR results were shown in Figure 1A.

We next examined whether target gene responses were specific for aRA, compared with other isomeric retinoid compounds, such as 13-cis-RA, which is in current clinical use in neuroblastoma patients (Matthay et al, 1999). Competitive RT-PCR analysis confirmed that all eight target genes tested were upregulated by 13-cis-RA in a manner similar to aRA in $\mathrm{BE}(2)-\mathrm{C}$ cells (Figure $1 \mathrm{~B}$ ). More specifically, RAR $\beta 2$, CYP26A1, CRBP1, RGS16, DUSP6, FLNB, PLAT, and RET were upregulated by 5.0-, 107.1-, 1.7-, $1.8-$, 1.9-, 4.5-, 2.6-, and 1.6-fold, respectively, 3 days after $10 \mu \mathrm{M}$ 13 -cis-RA treatment $(P<0.05$ in all cases).

\section{Regulation of RA target genes in neuroblastoma tumour tissue in vivo}

To assess whether the RA target genes identified in vitro were also modulated by RA in vivo, we treated homozygous MYCN transgenic mice with palpable abdominal neuroblastoma with 13-cis-RA $\left(0.72-1.43 \mathrm{mmol} 24 \mathrm{~h}^{-1}\right)$ or solvent control, and compared target gene expression by RT-PCR (Figure 1C). While no significant side effects were observed, the 5-day therapy with 13-cis-RA did not reduce tumour size, when compared with solvent-treated mice. Competitive RT - PCR showed that 13-cis-RA upregulated the expression of $\operatorname{RAR} \beta 2$ by $7.6 \pm 0.8$-fold, which was comparable to the in vitro data. In contrast, CYP26A1 was induced by only $2.0 \pm 0.3$-fold $(P<0.05)$, compared to induction by more than 100-fold in 13-cis-RA-treated BE(2)-C cells in vitro. Other RA target genes which were also upregulated in vivo included CRBP1 by $1.8 \pm 0.1$-fold, DUSP6 by $2.5 \pm 0.5$-fold, and PLAT by $1.9 \pm 0.2$ fold $(P<0.05)$. However, 13 -cis-RA did not significantly modulate RET, RGS16, FLNB, and EGR1 expression in the tissue samples from tumour-bearing animals treated for 5 days.

\section{Retinoid responsiveness of specific RA target genes correlates with the phenotypic retinoid response in neuroblastoma, lung and breast cancer cells}

We next asked whether the patterns of change in RA target gene expression seen in neuroblastoma cells were shared by other cancer types. RA-sensitive lung (Calu6) and breast (T47D) cancer cell lines, and RA-resistant lung (SK-MES-1) and breast (MDAMB-231) cancer cells were treated with control or $10 \mu \mathrm{M}$ aRA for 1 , 24 h, 3 or 7 days. Competitive RT - PCR was carried out to assess changes in the expression of the 13 target genes with cDNA from three independent cell culture and treatment experiments. As shown in Table 3 and Figure $1 \mathrm{~A}$ and D, four of 12 target genes (RAR $\beta 2$, CYP26A1, CRBP1, and RGS16) were upregulated by more than two-fold in both the RA-sensitive lung and breast cancer cells. However, RGS16, RAR $\beta 2$, and CYP26A1 were not detectable and not RA inducible in both of the RA-resistant breast and lung cancer cells. DUSP6 was induced in T47D cells only, while EGR1 was upregulated in Calu6 cells only. Surprisingly, RET and PLAT were downregulated by 10 - and 2.2 -fold in RA-sensitive Calu- 6 lung cancer cells, whereas both target genes were upregulated in both neuroblastoma cell lines. In contrast, ATP7A, DLK1, CRBP1, and SMAD3 were not modulated by aRA in RA-sensitive lung and breast cancer cells. 

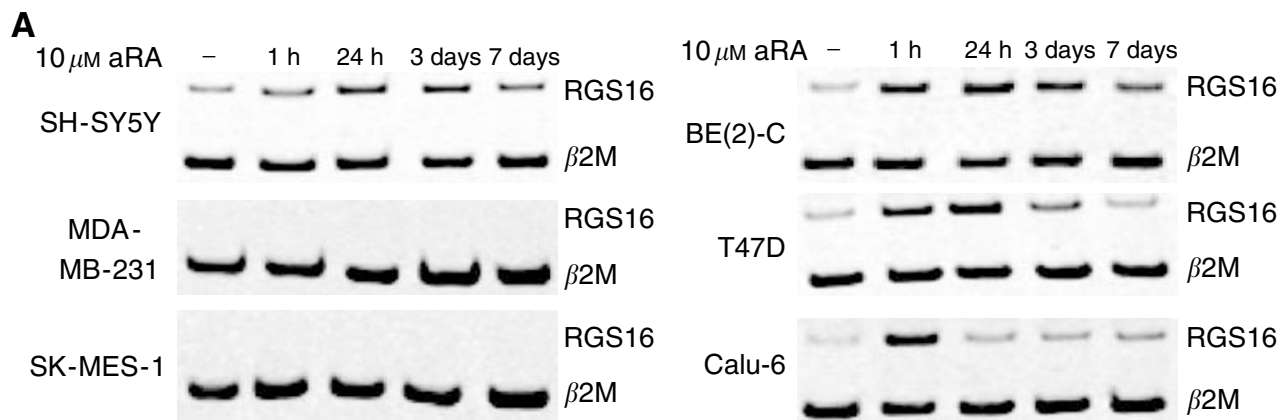

B

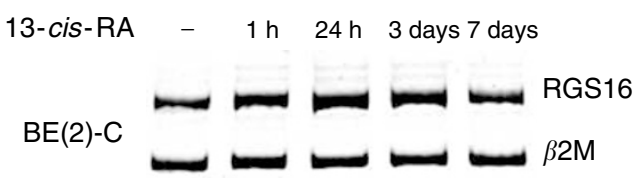

C
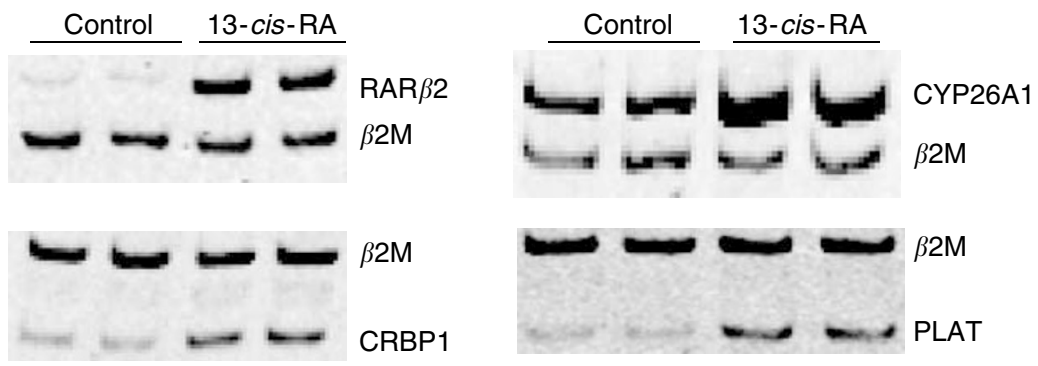

D
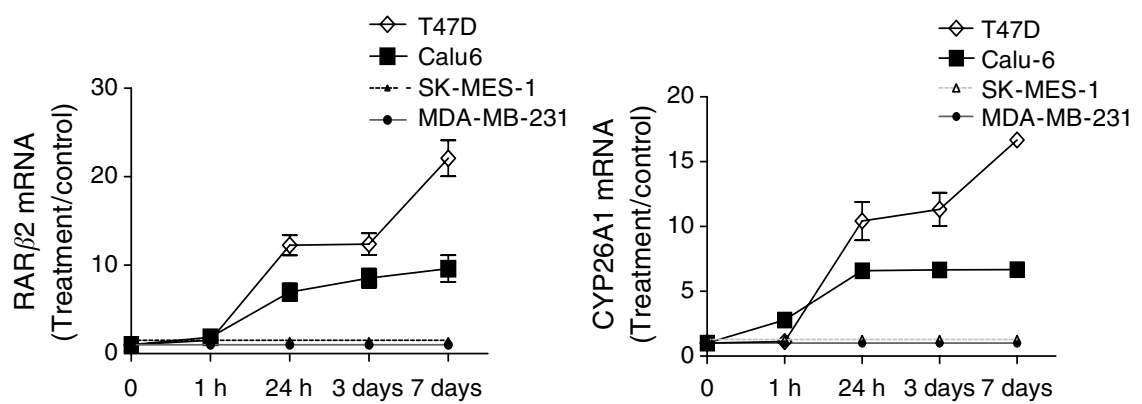

Figure I Induction of target gene expression by RA in neuroblastoma (SH-SY5Y, BE(2)-C), lung (SK-MES-I, Calu-6) and breast (MDA-MB-23I, T47D) cancer cell lines, and neuroblastoma tissues. cDNA samples from cultured cells treated with $10 \mu \mathrm{M}$ aRA $(\mathbf{A}$ and $\mathbf{D})$, or I3-cis-RA (B) or solvent control at various time points, and duplicate cDNA samples from neuroblastoma arising in MYCN transgenic mice treated with I3-cis-RA or control (C) were subjected to independent competitive RT-PCR analyses using trans-intron PCR primers, together with housekeeping gene $\beta 2 M$ primers. An equal aliquot of PCR product was then electrophoretically size-fractionated on a polyacrylamide gel as shown $(\mathbf{A}, \mathbf{B}$ and $\mathbf{C})$. Fold induction of a target gene by RA in RA-treated samples was calculated by ascribing the ratio between the level of expression of a target gene and that of $\beta 2 \mathrm{M}$ as 1.0 for control-treated samples (D).

\section{DNA demethylation and histone acetylation partially restore RAR $\beta 2$ and CYP26A1 gene expression in RA-resistant breast, but not lung cancer cells}

We observed a close correlation between the expression pattern of $\operatorname{RAR} \beta 2$ and CYP26A1 in response to retinoid (Tables 2 and 3 ). This led us to hypothesise that these two target genes may have a common mechanism of transcriptional repression in RA-resistant cells. To determine whether DNA methylation of regulatory elements resulted in the repression of $\operatorname{RAR} \beta 2$ and CYP26A1, RA-resistant lung and breast cancer cells were treated with the demethylating agent, aza-CdR. Treatment of cells with $0.1,1.0$, and $10 \mu \mathrm{M}$ aza-CdR, in combination with $10 \mu \mathrm{M}$ aRA for 3 days, did not restore RAR $\beta 2$ and CYP26A1 expression in the aRA-resistant cells when analysed by competitive RT-PCR. Minimally expressed genes may be out-competed by the $\beta 2 \mathrm{M}$ gene in a competitive RT-PCR (Freeman et al, 1999). Therefore, RAR $\beta 2$ and CYP26A1 expression was further analysed by noncompetitive RT-PCR. In noncompetitive RT - PCR, we detected weak RAR $\beta 2$ and CYP26A1 (Figure 2A) expression in the RA-resistant breast cancer cell line, MDA-MB-231, after treatment with aza-CdR and aRA, in a dosedependent manner. However, both RAR $\beta 2$ and CYP26A1 (Figure 2A) expression levels in treated MDA-MB-231 cells were relatively low compared to the levels seen in RA-sensitive lung cancer-positive control cells, Calu- 6 , also treated with $10 \mu \mathrm{M}$ aRA for 3 days. CYP26A1 and RAR $\beta 2$ transcripts were not detected by either competitive or noncompetitive RT - PCR in the RA-resistant lung cancer cell line. These data indicate that DNA methylation is one factor contributing to the repression of $\operatorname{RAR} \beta 2$ and CYP26A1 transcription in MDA-MB-231 cells. However, demethylation was insufficient to derepress transcription to a level equivalent to that seen in a RA-sensitive cell line, for both RAR $\beta 2$ and CYP26A1.

Since the level of histone acetylation, and conversely deacetylation, can influence gene transcription, the effect of the histone 
Table 3 Target gene fold induction by aRA in lung and breast cancer cells treated with $10 \mu \mathrm{M}$ aRA

\begin{tabular}{|c|c|c|c|c|c|c|c|c|c|}
\hline \multirow[b]{2}{*}{ Gene description } & \multirow[b]{2}{*}{ GeneBank accession } & \multicolumn{4}{|c|}{ Calu6 (SK-MES-I) } & \multicolumn{4}{|c|}{ T47D (MDA-MB-23I) } \\
\hline & & I h & $24 \mathrm{~h}$ & 3 days & 7 days & I h & $24 \mathrm{~h}$ & 3 days & 7 days \\
\hline RGS 16 & AA453774 & $2.1(-)$ & $1.4(-)$ & $1.6(-)$ & $1.4(-)$ & $3.2(-)$ & $2.7(-)$ & $1.7(-)$ & $1.4(-)$ \\
\hline $\mathrm{RAR} \beta 2$ & AA419238 & $1.5(-)$ & $7.5(-)$ & $8.6(-)$ & $9.8(-)$ & $1.2(-)$ & $12(-)$ & $13(-)$ & $22(-)$ \\
\hline CYP26AI & R5 I02I & $2.7(-)$ & $7.0(-)$ & $7.1(-)$ & $7.1(-)$ & I.I (-) & || (-) & || (-) & $17(-)$ \\
\hline CRBPI & AA700832 & I.I (I.5) & $1.4(1.6)$ & $1.5(1.7)$ & $2.0(2.0)$ & $1.5(1.5)$ & $2.5(1.6)$ & $2.7(1.9)$ & $5.3(2.0)$ \\
\hline ATP7A & AA23614I & I.I (0.9) & I.I (0.8) & $0.9(1.0)$ & $1.3(1.0)$ & I.I (1.0) & I.I (I.I) & $0.9(1.2)$ & $1.3(1.1)$ \\
\hline DUSP6 & AA630374 & I.I (I.I) & $1.3(1.1)$ & $1.4(1.1)$ & $1.4(1.3)$ & I.I (0.9) & $1.4(1.0)$ & $1.6(1.0)$ & $2.0(0.9)$ \\
\hline EGRI & AA486533 & $4.0(-)$ & $0.8(-)$ & $1.5(-)$ & $1.5(-)$ & $-(-)$ & $-(-)$ & $-(-)$ & $-(-)$ \\
\hline RET & $H 24956$ & $1.0(0.9)$ & $0.6(0.8)$ & $0.1(1.0)$ & $0.1(0.9)$ & $1.5(-)$ & $1.6(-)$ & $1.6(-)$ & $1.6(-)$ \\
\hline PLAT & AA447797 & $1.0(0.9)$ & $1.0(1.0)$ & $0.5(1.0)$ & $0.5(0.8)$ & $0.8(0.9)$ & $1.5(1.0)$ & $1.6(0.8)$ & $1.5(0.9)$ \\
\hline FLNB & AA486239 & $1.0(1.0)$ & $1.2(1.2)$ & $1.7(1.1)$ & $1.7(1.0)$ & I.I (I.I) & I.I (I.3) & $1.3(1.0)$ & $1.2(1.0)$ \\
\hline DLKI & AA576I 29 & $-(-)$ & $-(-)$ & $-(-)$ & $-(-)$ & $1.0(-)$ & $0.9(-)$ & $0.7(-)$ & $0.8(-)$ \\
\hline SMAD3 & W72201 & $1.0(1.0)$ & $1.0(1.0)$ & $1.0(1.0)$ & $1.0(1.0)$ & I.I ( 1.0$)$ & $1.1(1.0)$ & $1.0(1.0)$ & $1.0(0.9)$ \\
\hline
\end{tabular}

Calu6 and T47D cells are RA sensitive, while SK-MES-I and MDA-MB-23I are RA resistant. (-) represents undetectable by PCR.

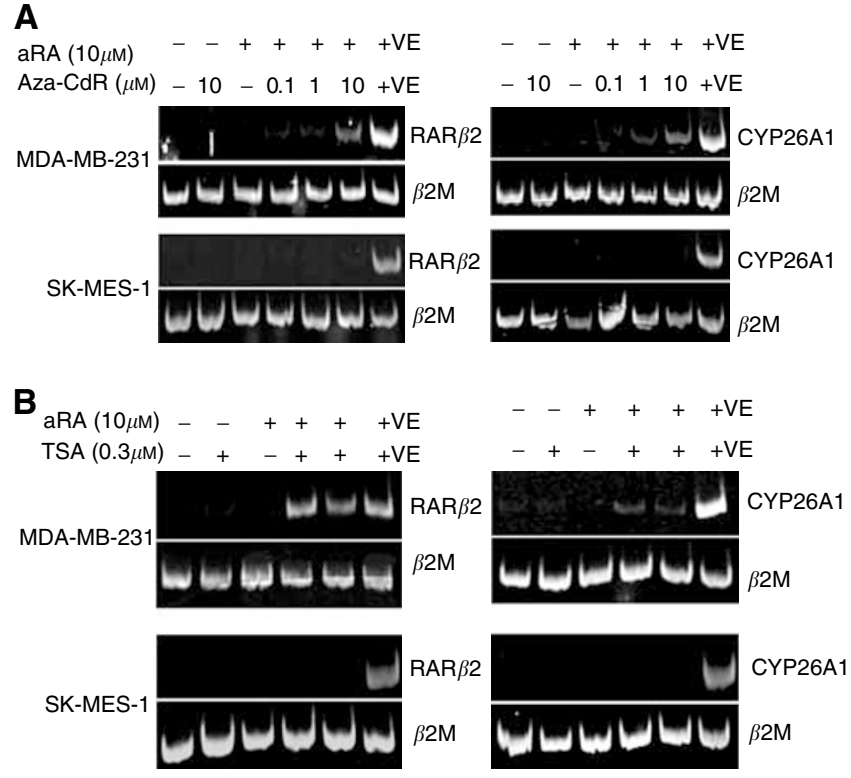

Figure 2 The effect of demethylation and acetylation on aRA-induced RAR $\beta 2$ and CYP26AI expression in RA-resistant cancer cells. RAR $\beta 2$ and CYP26AI expression was determined by noncompetitive RT-PCR, with expression of housekeeping gene $\beta 2 M$ as an internal control. (A) MDAMB-23 I and SK-MES- I cells were treated with control, aRA, and/or various concentration of aza-CdR for 3 days. (B) The cells were treated with control, aRA, and/or $0.3 \mu \mathrm{M}$ TSA for 3 days. The last lane of each gel $(+\mathrm{VE})$ contained a positive control from RA-sensitive Calu-6 cells treated with $10 \mu \mathrm{M}$ aRA for 3 days.

deacetylase (HDAC) inhibitor, TSA on RAR $\beta 2$ and CYP26A1 expression in RA-resistant lung and breast cancer cells was investigated. Cells were treated with $0.3 \mu \mathrm{m}$ TSA in combination with aRA (Sirchia et al, 2002) for $24 \mathrm{~h}$ and 3 days, and analysed for RAR $\beta 2$ and CYP26A1 (Figure 2B) expression. CYP26A1 and $\operatorname{RAR} \beta 2$ transcripts were detected in MDA-MB-231 cells after treatment with TSA and aRA, yet at a much lower level than in RA-treated Calu-6-positive control cells. CYP26A1 and RAR $\beta 2$ transcripts were not detected in SK-MES-1 cells. These observations together with the data from the demethylation experiments suggest that SK-MES-1 and MDA-MB-231 cells have different mechanisms of transcriptional repression for $\operatorname{RAR} \beta 2$ and CYP26A1, despite having a similar pattern of resistance to RA-induced transcription.

\section{Liganded RAR $\beta 2$ regulates RA-induced CYP26A1 expression}

We next examined whether restoring $\operatorname{RAR} \beta 2$ expression could restore CYP26A1 transcription in RA-resistant cells. MDA-MB-231 and SK-MES-1 cells were transiently transfected with a RAR $\beta 2$ cDNA expression vector or empty vector control, and treated with $10 \mu \mathrm{M}$ aRA for $24 \mathrm{~h}$ or 3 days following transfection (Figure $3 \mathrm{~A}$ and B). Results from a noncompetitive RT-PCR demonstrated a twofold increase in CYP26A1 levels in the RAR $\beta 2$-transfected MDAMB-231 cells, compared to cells transfected with the empty vector (Figure 3A). However, the SK-MES- 1 cells transfected with RAR $\beta 2$ did not demonstrate retinoid-inducible CYP26A1 expression (Figure 3B).

To further examine the possibility that $\mathrm{RAR} \beta 2$ directly regulated CYP26A1 transcription in RA-sensitive cells, SH-SY5Y cells were transiently transfected with an RAR $\beta 2$ cDNA expression vector or empty vector. Reverse transcription-polymerase chain reaction showed that RAR $\beta 2$ plasmid transfection upregulated RAR $\beta 2$ gene expression by about eight-fold, yet CYP26A1 expression was still undetectable, without RA treatment. In a separate experiment, SH-SY5Y cells, Calu-6 lung and T47D breast cancer cells were transiently transfected with scrambled siRNA or specific RAR $\beta 2$ siRNA and then treated with $10 \mu \mathrm{m}$ aRA for $48 \mathrm{~h}$. The RAR $\beta 2$ siRNA reduced RA-induced RAR $\beta 2$ transcription by about $80 \%$ in three independent transfection experiments (Figure 3C). Retinoic acid-induced CYP26A1 expression was downregulated by RAR $\beta 2$ siRNA by $36.47 \pm 6.57 \%$ in SH-SY-5Y cells, $39.85 \pm 5.38 \%$ in Calu- 6 cells, and $24 \pm 6.5 \%$ in T47D cells $(P<0.001)$. Since both RAR $\beta 2$ and CYP26A1 gene expression was not detectable without aRA treatment in all aRA-sensitive and -resistant cancer cells tested, we did not carry out RAR $\beta 2$ siRNA transfection experiments without treatment with aRA. These results indicated that RA-induced CYP26A1 expression required retinoid liganded $\operatorname{RAR} \beta 2$, in RA-sensitive and some RA-resistant cells. Interestingly, transient transfection of RAR $\beta 2$ siRNA did not repress other RA-inducible gene expression (DUSP6, RGS16, FLNB, CRBP1, RET, PLAT).

\section{Synchronous RA-induced expression of DUSP6 and RGS16} is required for the antiproliferative effect of RA

DUSP6 and RGS16 are both inhibitors involved in the ERK MAPK signalling pathway, RGS16 at the level of Ras G proteins (Buckbinder et al, 1997; Chen et al, 2001) and DUSP6 at the level of ERK proteins (Groom et al, 1996; Sah et al, 2002; Nakagawa et al, 2003). Since the growth inhibitory effect of RA is at least partly due to reduction in ERK phosphorylation (Nakagawa et al, 2003), we examined the hypothesis that RA-induced proliferative 
A

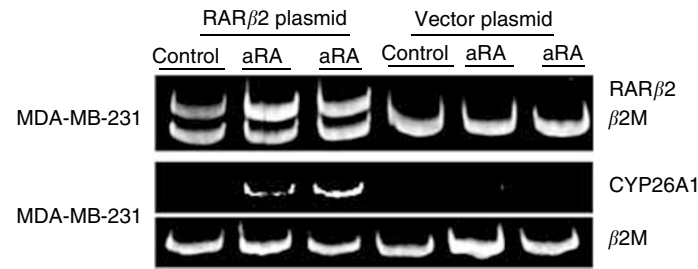

B

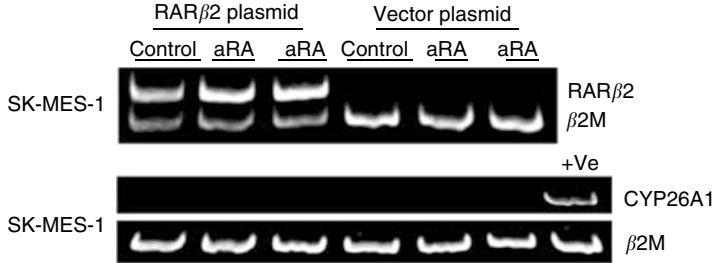

C
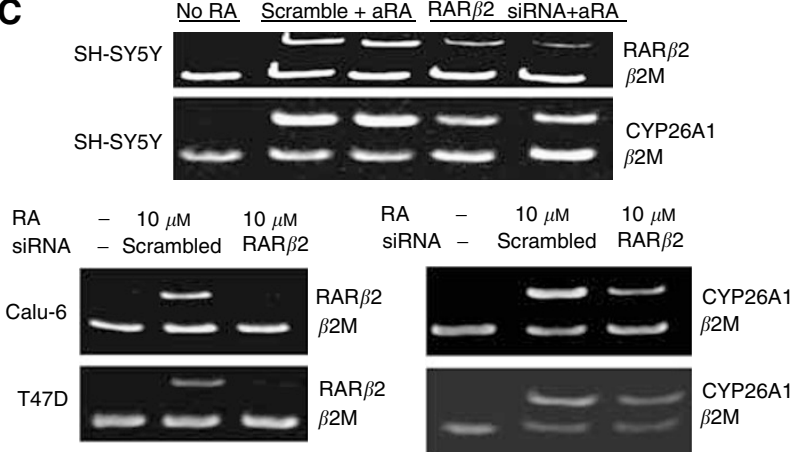

Figure 3 Liganded RAR $\beta 2$ modulates CYP26AI expression. RAR $\beta 2$ gene expression was analysed with the standard competitive RT-PCR, and CYP26Al expression was analysed by competitive RT-PCR in RAsensitive SH-SY5Y, Calu-6, and T47D cells and noncompetitive RT-PCR in RA-resistant cells, with housekeeping gene $\beta 2 \mathrm{M}$ as an internal control. (A B) CYP26AI transcription was determined in MDA-MB-23I (A) or SKMES-I cells (B) transiently transfected with RAR $\beta 2$ cDNA plasmid or vector plasmid and treated with control or $10 \mu \mathrm{M}$ aRA for $24 \mathrm{~h}$ (Lanes 2 and 5) or 3 days (lanes 3 and 6). Last lane in (B) contained the positive control from Calu- 6 cells treated with $10 \mu \mathrm{M}$ aRA for 3 days. (C) RAR $\beta 2$ and CYP26AI expression was analysed in SH-SY5Y, Calu-6, and T-47D cells transfected with scramble siRNA or RAR $\beta 2$ siRNA and treated with $10 \mu \mathrm{M}$ aRA for $48 \mathrm{~h}$.

arrest was dependent on increased expression of DUSP6 or RGS16. Three DUSP6 and three RGS16 siRNA duplexes were in vitro transcribed, and transfected into SH-SY5Y cells, followed by $10 \mu \mathrm{M}$ aRA treatment. Competitive RT-PCR showed that the DUSP6 siRNA targeting AAGTGCGGAATTGGTTAATAC, and the RGS16 siRNA targeting AACAAGGCAGAAAAGGATCCT, were the most efficient siRNAs at reducing the expression of each gene (Figure 4A). These siRNAs were, therefore, chosen in all further experiments for protein and cell proliferation studies. As shown in Figure $4 \mathrm{~B}, 48 \mathrm{~h}$ of treatment with $10 \mu \mathrm{m}$ aRA induced RGS16 protein by two-fold, and DUSP6 protein more dramatically from only just detectable, compared with solvent control. At the same time point, RGS16 siRNAs effectively counteracted RA-responsive RGS16 overexpression, while DUSP6 siRNA abolished RA-responsive DUSP6 induction.

To determine the roles of DUSP6 and RGS16 in MAPK ERK dephosphorylation, cell lysates from SH-SY5Y cells transfected with scrambled, DUSP6, and/or RGS16 siRNAs with or without $10 \mu \mathrm{M}$ aRA treatment for $60 \mathrm{~h}$ were subjected to ERK and phosphorylated ERK immunoblot. Without aRA intervention, DUSP6 and/or RGS16 siRNA transfection did not have an effect on ERK phosphorylation. Compared with scrambled siRNA counterparts, DUSP6 siRNA increased ERK phosphorylation by

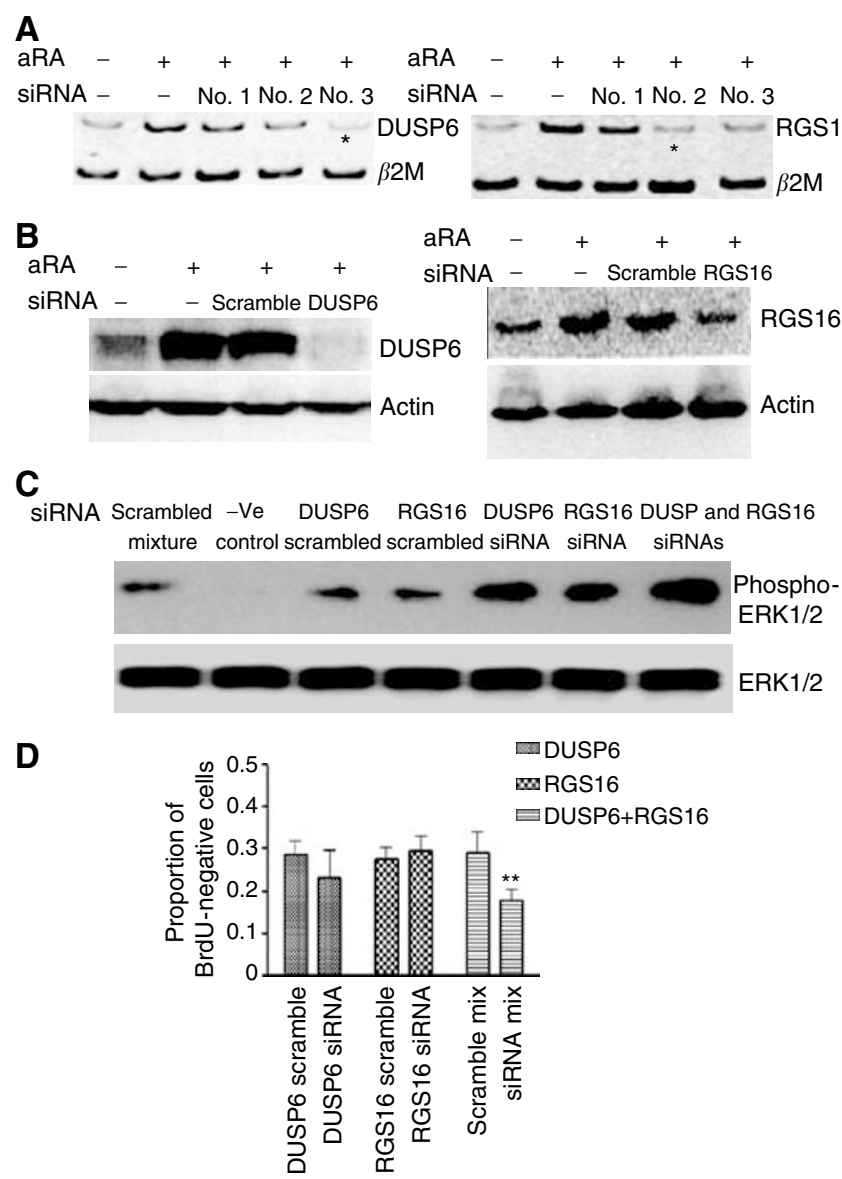

Figure 4 Synchronous expression of both DUSP6 and RGSI6 contributed to RA-induced growth inhibition. (A) DUSP6 and RGSI6 gene expression was analysed with competitive RT-PCR with the housekeeping gene $\beta 2 \mathrm{M}$ as an internal control with samples from $\mathrm{SH}$ SY5Y cells transfected with DUSP6 or RGSI 6 siRNA or scrambled siRNA and treated with $10 \mu \mathrm{M}$ aRA for $48 \mathrm{~h}$. * Indicates the siRNAs of choice for protein and functional studies. (B) DUSP6 and RGSI6 protein was analysed by Western blot with samples from $\mathrm{SH}-\mathrm{SY} 5 Y$ cells transfected with scrambled siRNA, DUSP6, or RGSI6 siRNA and treated with $10 \mu \mathrm{M}$ aRA or control solvent for $48 \mathrm{~h}$. $\beta$-Actin protein was used as a loading control. (C) Phosphorylated ERKI/2 was analysed by Western blot with samples from SH-SY5Y cells transfected with scrambled, DUSP6, RGSI6 siRNA, or siRNA combinations and treated with $10 \mu \mathrm{M}$ aRA for $60 \mathrm{~h}$. Total ERKI/2 protein was used as a loading control. (D) BrdU incorporation into proliferating cells was analysed in $\mathrm{SH}-\mathrm{SY} 5 \mathrm{Y}$ cells after transfection with scrambled or target gene siRNAs plus treatment with $10 \mu \mathrm{M}$ aRA for $64 \mathrm{~h}$. BrdU-positive cells treated with vehicle solvent and transfected with scrambled siRNA were artificially set as 100\%. Error bar represented standard error. ***indicated statistical significant difference $(P<0.05)$.

about 2.5-fold, while RGS16 siRNA induced ERK phosphorylation by 1.4-fold (Figure 4C). When cells were cotransfected with siRNAs against DUSP6 and RGS16, we observed an additive effect on ERK phosphorylation of a further 1.6-fold compared with DUSP siRNA alone, or four-fold compared with scrambled siRNA mixture (Figure 4C). All siRNAs did not show any effect on total ERK protein expression.

In the cell proliferation studies, BrdU incorporation by SH-SY5Y cells transfected with scrambled siRNA was decreased after $60 \mathrm{~h}$ of aRA treatment, compared with solvent control. We did not observe a significant effect of transfection of DUSP6 siRNA or RGS16 siRNA alone, compared with scrambled siRNA, on BrdU uptake (Figure 4D). In contrast, transfection of a combination of DUSP6 and RGS16 siRNAs reduced the proportion of BrdU-negative cells 
due to aRA treatment, and reduced the effect of aRA on BrdU incorporation by more than $40 \%(P<0.05)$. These findings indicated that combined inhibition of the MAPK at two levels mediated the retinoid effects on cell proliferation. As DUSP6 protein was hardly detectable without RA treatment, the effect of siRNA in the cell proliferation assay was not carried out without RA treatment.

\section{DISCUSSION}

In this study, we used cDNA microarray to identify 31 RAregulated target genes shared by two RA-sensitive neuroblastoma cells lines, and then evaluated the relevance of RA-induced changes in target gene expression to the RA anticancer effect. Our data suggest that RA-induced changes in the expression of RAR $\beta 2$, CYP26A1, CRBP1, RGS16, DUSP6, and EGR1 may be necessary for the retinoid anticancer signal in neuroblastoma, breast and/or lung cancer cells. However, we found little evidence for a direct signalling relationship among these genes, except that RA-induced CYP26A1 expression is partly modulated by RAR $\beta 2$. Our data showed clearly that exogenous overexpression of RAR $\beta 2$ did not induce CYP26A1 expression in the absence of RA ligand, even in RA-sensitive cancer cells. However, forced overexpression of RAR $\beta 2$ activated CYP26A1 transcription in RA-resistant breast cancer cells when the RAR $\beta 2$ ligand, RA, was added. Consistently, knocking down of RA-induced RAR $\beta 2$ expression partly blocked RA-induced CYP26A1 expression across RA-sensitive neuroblastoma, lung and breast cancer cells. This inter-relationship is in agreement with earlier studies, which showed that exogenous RAR $\beta 2$ overexpression in RA-resistant HCT- 116 colon cancer cells partially restored RA-induced CYP26A1 expression (Sonneveld et al, 1998). Our data indicate that the close link between RAR $\beta 2$ and CYP26A1 expression patterns may be explained by the dependence of CYP26A1 expression on liganded RAR $\beta 2$. Additionally, we found that 13-cis-RA only marginally upregulated CYP26A1 expression in neuroblastoma tissues in vivo, while inducing $\operatorname{RAR} \beta 2$ expression to a similar extent as in vitro. This observation suggests that there may be other mechanisms limiting the extent to which 13-cis-RA liganded RAR $\beta 2$ can induce CYP26A1 in vivo.

DNA methylation and histone deacetylation of the RAR $\beta 2$ gene promoter region have been proposed to result in $\operatorname{RAR} \beta 2$ silencing and RA resistance (Sirchia et al, 2002; Suh et al, 2002). Our study confirmed these findings and found, for the first time, that both DNA methylation and HDAC contribute to CYP26A1 silencing in MDA-MB-231 cells. In contrast, neither DNA demethylation nor the HDAC inhibitor had any effect on RA-induced CYP26A1 or $\operatorname{RAR} \beta 2$ expression in SK-MES-1 cells. The RAR $\beta 2$ gene promoter has been shown to be hypermethylated and hypoacetylated in SK-MES-1 cells, and to be resistant to DNA demethylation and histone acetylation (Suh et al, 2002). The CYP26A1 gene promoter may also have been resistant to demethylating or acetylating agents due to as yet undefined mechanisms.

This study, for the first time, identified DUSP6 and RGS16 as novel RA target genes, and found that synchronous knock down of RA-induced DUSP6 and RGS16 expression synergistically/additively increased MAPK ERK phosphorylation and partly blocked RA-induced growth inhibition, although decreased expression of either protein alone was insufficient to effect cell proliferation. DUSP6 encodes a dual-specificity phosphatase specific for ERK (Groom et al, 1996), a key effector MAPK involved in the RAS-GTP signal transduction pathway (Hunter, 1995; Becker, 2004). DUSP6 dephosphorylates activated ERK and blocks the growth-stimulatory signals (Furukawa et al, 2003; Furukawa and Horii, 2004). RGS16, on the other hand, acts as a mechanism for p53 to exert cellular growth control and acts as a negative feedback regulator in response to mitogenic signals (Buckbinder et al, 1997). RGS16 inhibits ERK activation upstream of the RAS-RAF-MEKERK pathway by enhancing GTPase-activating protein function and inactivating RAS-GTP (Buckbinder et al, 1997; Chen et al, 2001). Various inhibitors of RAS-RAF-MEK-ERK signalling pathway have been proven to inhibit cancer cell growth and survival, and are already in phase II clinical trials for the treatment of various cancers (Sebolt-Leopold and Herrera, 2004). DUSP6 and RGS16 transcriptional upregulation inhibits tumour cell proliferation and ERK phosphorylation, and serves as a negative regulatory mechanism to prevent further ERK phosphorylation. Our data therefore indicates that RA-induced upregulation of DUSP6 and RGS16 inhibits tumour cell proliferation, through acting on two levels of RAS-RAF-MEK-ERK signalling pathway and eventually synergistically reducing ERK phosphorylation.

In conclusion, we have demonstrated both classical and novel mechanisms by which RA-regulated target gene expression patterns may relate to the retinoid anticancer signal. Our findings have confirmed the importance of previously recognised signalling molecules such as RAR $\beta 2$, and have identified novel roles for MAPK signal inhibitory proteins DUSP6 and RGS16 in mediating the retinoid anticancer effect.

\section{ACKNOWLEDGEMENTS}

This work was supported by National Health and Medical Research Council of Australia, Cancer Council NSW, and Sydney Children's Hospital Foundation. Children's Cancer Institute Australia for Medical Research is affiliated to the University of New South Wales and Sydney Children's Hospital.

\section{REFERENCES}

Altucci L, Gronemeyer H (2001) The promise of retinoids to fight against cancer. Nat Rev Cancer 1: 181-193

Balmer JE, Blomhoff R (2002) Gene expression regulation by retinoic acid. J Lipid Res 43: $1773-1808$

Becker EW (2004) Relevance of the kinetic equilibrium of forces to the control of the cell cycle by Ras proteins. Biol Chem 385: $41-47$

Bordow SB, Norris MD, Haber PS, Marshall GM, Haber M (1998) Prognostic significance of MYCN oncogene expression in childhood neuroblastoma. J Clin Oncol 16: 3286-3294

Boussioutas A, Li H, Liu J, Waring P, Lade S, Holloway AJ, Taupin D, Gorringe K, Haviv I, Desmond PV, Bowtell DD (2003) Distinctive patterns of gene expression in premalignant gastric mucosa and gastric cancer. Cancer Res 63: 2569-2577

Buckbinder L, Velasco-Miguel S, Chen Y, Xu N, Talbott R, Gelbert L, Gao J, Seizinger BR, Gutkind JS, Kley N (1997) The p53 tumor suppressor targets a novel regulator of G protein signaling. Proc Natl Acad Sci USA 94: $7868-7872$

Burkhart CA, Cheng AJ, Madafiglio J, Kavallaris M, Mili M, Marshall GM, Weiss WA, Khachigian LM, Norris MD, Haber M (2003) Effects of MYCN antisense oligonucleotide administration on tumorigenesis in a murine model of neuroblastoma. J Natl Cancer Inst 95: 1394-1403

Chambon P (1996) A decade of molecular biology of retinoic acid receptors. FASEB J 10: $940-954$

Chen C, Wang H, Fong CW, Lin SC (2001) Multiple phosphorylation sites in RGS16 differentially modulate its GAP activity. FEBS Lett 504: $16-22$

Cheung B, Hocker JE, Smith SA, Norris MD, Haber M, Marshall GM (1998) Favorable prognostic significance of high-level retinoic acid receptor beta expression in neuroblastoma mediated by effects on cell cycle regulation. Oncogene 17: $751-759$ 
Cheung B, Yan J, Smith SA, Nguyen T, Lee M, Kavallaris M, Norris MD, Haber M, Marshall GM (2003) Growth inhibitory retinoid effects after recruitment of retinoid $\mathrm{X}$ receptor beta to the retinoic acid receptor beta promoter. Int J Cancer 105: 856-867

Elbashir SM, Harborth J, Lendeckel W, Yalcin A, Weber K, Tuschl T (2001) Duplexes of 21-nucleotide RNAs mediate RNA interference in cultured mammalian cells. Nature 411: 494-498

Evans TR, Kaye SB (1999) Retinoids: present role and future potential. Br J Cancer 80: $1-8$

Freeman WM, Walker SJ, Vrana KE (1999) Quantitative RT-PCR: pitfalls and potential. Biotechniques 26: $112-122$

Freemantle SJ, Spinella MJ, Dmitrovsky E (2003) Retinoids in cancer therapy and chemoprevention: promise meets resistance. Oncogene 22: $7305-7315$

Furukawa T, Horii A (2004) Molecular pathology of pancreatic cancer: in quest of tumor suppressor genes. Pancreas 28: 253-256

Furukawa T, Sunamura M, Motoi F, Matsuno S, Horii A (2003) Potential tumor suppressive pathway involving DUSP6/MKP-3 in pancreatic cancer. Am J Pathol 162: 1807-1815

Groom LA, Sneddon AA, Alessi DR, Dowd S, Keyse SM (1996) Differential regulation of the MAP, SAP and RK/p38 kinases by Pyst1, a novel cytosolic dual-specificity phosphatase. EMBO J 15: $3621-3632$

Houle B, Rochette-Egly C, Bradley WE (1993) Tumor-suppressive effect of the retinoic acid receptor beta in human epidermoid lung cancer cells. Proc Natl Acad Sci USA 90: 985-989

Hunter T (1995) Protein kinases and phosphatases: the yin and yang of protein phosphorylation and signaling. Cell 80: 225-236

Matthay KK, Villablanca JG, Seeger RC, Stram DO, Harris RE, Ramsay NK, Swift P, Shimada H, Black CT, Brodeur GM, Gerbing RB, Reynolds CP (1999) Treatment of high-risk neuroblastoma with intensive chemotherapy, radiotherapy, autologous bone marrow transplantation, and 13cis-retinoic acid. Children's Cancer Group. N Engl J Med 341: 1165 - 1173

Nakagawa S, Fujii T, Yokoyama G, Kazanietz MG, Yamana H, Shirouzu K (2003) Cell growth inhibition by all-trans retinoic acid in SKBR-3 breast cancer cells: involvement of protein kinase $\mathrm{C}$ alpha and extracellular signal-regulated kinase mitogen-activated protein kinase. Mol Carcinog 38: $106-116$

Nguyen T, Hocker JE, Thomas W, Smith SA, Norris MD, Haber M, Cheung B, Marshall GM (2003) Combined RAR alpha- and RXR-specific ligands overcome N-myc-associated retinoid resistance in neuroblastoma cells. Biochem Biophys Res Commun 302: $462-468$

Norris MD, Bordow SB, Marshall GM, Haber PS, Cohn SL, Haber M (1996) Expression of the gene for multidrug-resistance-associated protein and outcome in patients with neuroblastoma. $N$ Engl J Med 334: 231 - 238

Sah JF, Eckert RL, Chandraratna RA, Rorke EA (2002) Retinoids suppress epidermal growth factor-associated cell proliferation by inhibiting epidermal growth factor receptor-dependent ERK1/2 activation. J Biol Chem 277: $9728-9735$

Sebolt-Leopold JS, Herrera R (2004) Targeting the mitogen-activated protein kinase cascade to treat cancer. Nat Rev Cancer 4: 937-947

Sirchia SM, Ren M, Pili R, Sironi E, Somenzi G, Ghidoni R, Toma S, Nicolo G, Sacchi N (2002) Endogenous reactivation of the RARbeta2 tumor suppressor gene epigenetically silenced in breast cancer. Cancer Res 62: $2455-2461$

Sonneveld E, van den Brink CE, van der Leede BM, Schulkes RK, Petkovich M, van der Burg B, van der Saag PT (1998) Human retinoic acid (RA) 4hydroxylase (CYP26) is highly specific for all-trans-RA and can be induced through RA receptors in human breast and colon carcinoma cells. Cell Growth Differ 9: 629-637

Suh YA, Lee HY, Virmani A, Wong J, Mann KK, Miller Jr WH, Gazdar A, Kurie JM (2002) Loss of retinoic acid receptor beta gene expression is linked to aberrant histone $\mathrm{H} 3$ acetylation in lung cancer cell lines. Cancer Res 62: $3945-3949$

Sun SY, Lotan R (2002) Retinoids and their receptors in cancer development and chemoprevention. Crit Rev Oncol Hematol 41: $41-55$

Weiss WA, Aldape K, Mohapatra G, Feuerstein BG, Bishop JM (1997) Targeted expression of MYCN causes neuroblastoma in transgenic mice. EMBO J 16: 2985 - 2995 